LAWRENCE LIVERMORE NATIONAL LABORATORY
Hyperspectral Imaging of

Functional Patterns for

Disease Assessment and

Treatment Monitoring

Moinuddin Hassan, David Hattery, Abby Vogel, Victor Chernomordik, Stavros Demos, Karen Aleman, Richard Little, Robert Yarchoan Amir H Gandjbakhche

December 8, 2003

OSA Biomedical Optics Topical Meetings

Miami Beach, Florida, April 14-17, 2004 
This document was prepared as an account of work sponsored by an agency of the United States Government. Neither the United States Government nor the University of California nor any of their employees, makes any warranty, express or implied, or assumes any legal liability or responsibility for the accuracy, completeness, or usefulness of any information, apparatus, product, or process disclosed, or represents that its use would not infringe privately owned rights. Reference herein to any specific commercial product, process, or service by trade name, trademark, manufacturer, or otherwise, does not necessarily constitute or imply its endorsement, recommendation, or favoring by the United States Government or the University of California. The views and opinions of authors expressed herein do not necessarily state or reflect those of the United States Government or the University of California, and shall not be used for advertising or product endorsement purposes.

This work was performed under the auspices of the U.S. Department of Energy by University of California, Lawrence Livermore National Laboratory under Contract W-7405-Eng-48. 


\title{
Hyperspectral Imaging of Functional Patterns for Disease Assessment and Treatment Monitoring
}

\author{
David Hattery, Moinuddin Hassan \\ National Institutes of Health, Bethesda, MD 20892-0924 \\ ph. 301 594-0352, fax 301 480-2427, hattery@ieee.org, hassanm@mail.nih.gov \\ Stavros Demos \\ Lawrence Livermore National Laboratory, Livermore, CA 94551, demos1 @1lnl.gov \\ Karen Aleman, Richard Little, Robert Yarchoan \\ National Institutes of Health, Bethesda, MD 20892-1868, yarchoan@helix.nih.gov \\ Amir Gandjbakhche \\ National Institutes of Health, Bethesda, MD 20892-0924, amir@ helix.nih.gov
}

\begin{abstract}
We have designed and built a six-band multi-spectral NIR imaging system used in clinical testing on cancer patients. From our layered tissue model, we create blood volume and blood oxygenation images for patient treatment monitoring.

OCIS Codes: $170.0110,170.1470,170.3010,170.3660,170.3880,170.5280,170.6510,170.7050$
\end{abstract}

\section{Introduction}

Optical spectroscopic methods have great utility in molecular characterization of disease processes. Optical methods, however, have been primarily limited to in-vitro or cell culture studies. This is because strong scattering in tissue causes dispersion of the photons paths which results in poor localization and resolution. Not only does scattering change the depth of photon penetration, path length dispersion also makes spectroscopic reconstruction of analyte concentrations difficult since the path length, over which photons are subject to molecularly-specific absorption, changes.

Current methods for quantifying analyte concentration in tissue require accurate knowledge of tissue scattering, as well as defined source-detector separations. Researchers are employing devices ranging from simple fiber-optic probes, which employ a point source and a point detector, to source-detector arrays that multiplex and provide many such point sources and detectors. In general, these devices fail to provide enough measurements to form complete images without extremely complex, bulky probes that are difficult to use. Even then, the spatial resolution is not as high as can be obtained from a CCD imager.

We believe there is a need for an imaging system that provides quantitative contrast of analyte concentrations below the tissue surface. Such an imaging system would not need the absolute quantitative accuracy since disease status would be determined from patterns rather than absolute values. Such a system could be used to visualize and monitor the functional behavior of tumors in vivo without requiring invasive procedures or incurring patient discomfort.

We have designed and built a spectral imager that captures images at six wavelengths in the NIR $(700,750,800$, 850,900 , and $1000 \mathrm{~nm}$ ). The NIR wavelength light penetrates into tissue farther than other wavelengths due to tissue's low absorption in the NIR. To further increase the penetration of detected light, polarization filters in crosspolarized orientation are used to selectively filter photons that have only scattered a few times. Thus light scattered from shallow tissue, which generally corresponds to very short photon path lengths in tissue, are blocked whereas the deeper penetrating, more scattered photons depolarize and are detected.

The data from our imager are used to reconstruct local variations in the concentrations of analytes such as oxy- and deoxy-hemoglobin in tissue. This imager has been used in clinical testing on patients with Kaposi's Sarcoma (KS), a highly vascular skin tumor, at the start of, and at periodic intervals during anti-angiogenesis therapy.

\section{Theory}

In our model, we have two layers: the epidermis and the underlying dermis. As input to our model, we use absorption spectra for the analytes. Each of the band-pass filters on the imager has a full width at half max (fwhm) of $50 \mathrm{~nm}$. Before using the absorption data for reconstruction, the data must be convolved with the response function of the band-pass filters.

For light to reach the tumor it must pass through the epidermis. This transmission can be shown to be a function of the melanin in the epidermis and the epidermal thickness (1). We combine all other epidermal skin analytes into 
a single absorption variable $\mu_{a(\text { skin })}(\lambda)$ which is a function of wavelength (2). The dominant chromophores in that spectrum are lipids and water. A volume fraction model is used with those spectra to obtain an epidermis absorption coefficient. The epidermis is typically $0.1 \mathrm{~mm}$ thick which is approximately the mean scattering distance of photons in tissue. Scattering in tissue is typically very anisotropic with $g \approx 0.9$. This means that the few epidermal scatterings will typically be shallow angle and the transmission through the layer may be considered to be independent of scattering. Thus, Beer's law may be used for the intensity attenuation through this layer.

The underlying dermis is where the tumor and blood vessels are located. We may assume that the absorption of blood in tissue is a function of the volume fraction of oxy- and deoxy-hemoglobin, $V_{\text {oxy }}$ and $1-V_{o x y}$ respectively. The total blood absorption is $\mu_{a \text { (blood })}(\lambda)=V_{\text {oxy }} \mu_{a(\text { oxy })}(\lambda)+\left(1-V_{\text {oxy }}\right) \mu_{a(\text { deoxy })}(\lambda)$. The remaining, non-blood fraction is considered skin with $\mu_{a(\text { skin })}(\lambda)$ as used for the epidermis. The sub-epidermal layer may be assumed to be infinitely thick and the light passing from the epidermis, $I_{e p i}(\lambda)$, is no longer collimated due to epidermal scattering. The scattering is modeled using analytical solutions developed from a random walk model of photon migration in turbid media. The reflected intensity from the dermis is (3):

$$
I_{\text {ref }}(\lambda)=I_{e p i} \frac{1-e^{-\sqrt{24 \mu_{a(\text { derm })}(\lambda) / \mu_{s}^{\prime}(\lambda)}}}{\sqrt{24 \mu_{a(\text { derm })}(\lambda) / \mu_{s}^{\prime}(\lambda)}}
$$

where $\mu_{a(\text { derm })}(\lambda)=V_{\text {blood }} \mu_{a(\text { blood })}(\lambda)+\left(1-V_{\text {blood }}\right) \mu_{a(\text { skin })}(\lambda)$ and $\mu_{s}{ }^{\prime}(\lambda)$ is the transport-corrected scattering coefficient. Equation 1 is the integrated intensity over the entire plane for point illumination. In practice, we are looking at intensities at single pixels given illuminance over the entire plane. These are reversible by symmetry. We may then express the intensity detected by a camera as follows (4):

$$
I_{\text {detected }}(\lambda)=c I_{s}(\lambda) d(\lambda) 2 I_{\text {epi }}(\lambda) I_{\text {ref }}(\lambda)
$$

where $I_{s}(\lambda)$ is the source intensity and $d(\lambda)$ is the spectral response of the camera. The product of $d(\lambda)$ and $I_{s}(\lambda)$ is generally combined into a single calibration function for the imaging device. Besides the known spectra, equation 2 contains five variables, $V_{o x y}, V_{\text {blood }}, V_{m e l}, t$, and our intensity scaling factor, $c$. The six spectral bands of our imaging system allow for the reconstruction of up to six unknowns. For this work, we fixed the epithelial thickness at $0.1 \mathrm{~mm}$ and the volume fraction of melanin at 0.1 . The biggest source of error in Equation 2 is related to the change in $\mu_{s}^{\prime}$ as a function of wavelength. The change in path length is accounted for in Equation 1, but as $\mu_{s}^{\prime}$ decreases, the volume sampled by the photons increases. Since the tissue has physical structures with different biochemical makeup, changing the sampling volume as wavelength increases changes the actual concentrations of sampled analytes. This introduces an error into the analyte concentration values, so we will instead focus on spatial patterns in reconstructed analyte concentration and changes in those patterns as an indicator of disease status and progression.

\section{Results}

We have collected data from nearly 100 imaging sessions spanning two years. The following are two representative patients that have been imaged several times for whom we are only showing blood oxygen saturation levels. Figure 1 shows an image taken of patient 1 during treatment that indicates the patient still has a metabolically active tumor. This is indicated by generally low reconstructed blood oxygen-fraction in the tumor region; the tumor metabolic demand is exceeding supply and the tumor is hypoxic. More importantly, the blood oxygen contrast at the site of visible tumor activity is very distinct. After 8 more months of treatment, the tumors are still visible in the $700 \mathrm{~nm}$ image. The contrast in the blood oxygen image, however, has been greatly reduced indicating that the functional activity of the tumor is more similar to the surrounding tissue than it had been.

An image of patient 1 before treatment is shown in Figure 2 in which there is large blood oxygen-fraction contrast around the boundary of the tumor. After 5 months of treatment, however, the contrast has increased. Further, the tumor has two visible regions: the left where oxygen-fraction is elevated above the background and the right side where it is below the background.

\section{Conclusion and Future Directions}

Quantification of analyte concentrations in tissue requires a model to account for path length changes due to scattering, the scattering coefficient, and fixed source-detector separations. This makes it very difficult to obtain high spatial resolution images of analyte concentrations. By employing a multi-spectral NIR imaging system and a model of diffuse reflectance, images of relative analyte concentration may be obtained. This quantitative contrast may be used to visualize functional patterns associated with tumors. 

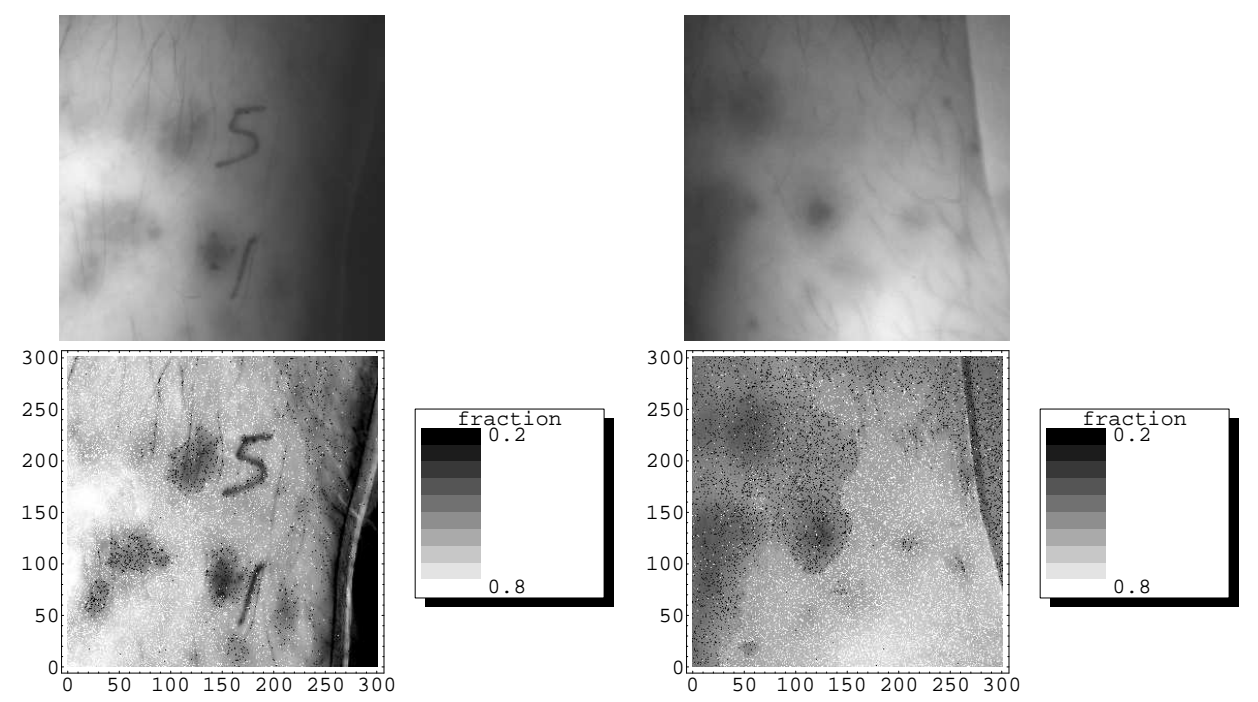

Figure 1: Patient 1, $700 \mathrm{~nm}$ images (top), blood oxygen-fraction images (bottom), during treatment (left) and 8 months later (right)
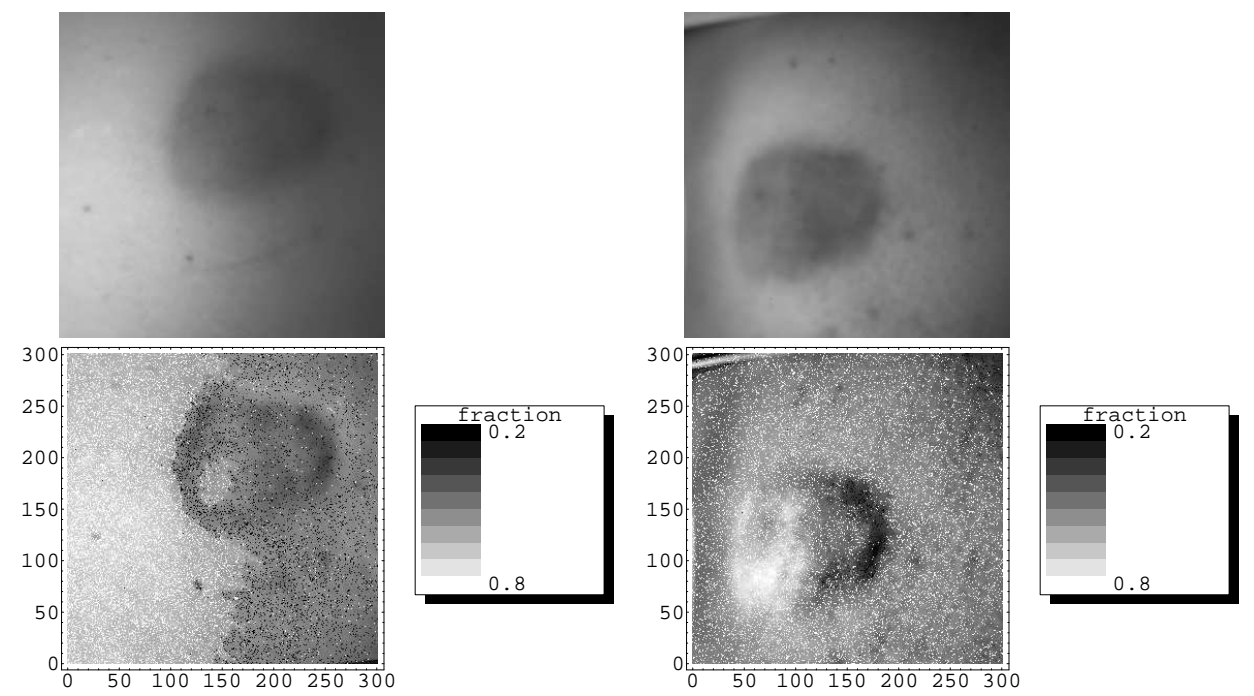

Figure 2: Patient 2, $700 \mathrm{~nm}$ images (top), blood oxygen-fraction images (bottom), before treatment (left) and 5 months (right)

Boundaries of KS tumor functional behavior have been imaged and changes during therapy have been observed. In some cases, the changes reduce contrast over the entire tumor which suggests that the tissue is returning to a more homogeneous, non-diseased state. In other cases, contrast within the tumor indicates that the disease progression is not uniform and some parts of the tumor are being effected more than others. The underlying cause of this contrast has not been fully understood and is an area for continued research. The different responses of patients, however, is potentially very useful information to physicians evaluating the response of patients to treatment.

\section{References}

[1] S. L. Jacques and D. J. McAuliffe, "The melanosome: threshold temperature for explosive vaporization and internal absorption coefficient during pulsed laser irradiation," Photochemistry and Photobiology 53, pp. 769$775,1991$.

[2] I. S. Saidi, S. L. Jacques, and F. K. Tittle, "Mie and Rayleigh Modeling of Visible-light Scattering in Neonatal Skin," Applied Optics 34(31), pp. 7410-7418, 1995.

[3] A. H. Gandjbakche and G. H. Weiss, "Random walk and diffusion-like models of photon migration in turbid media," in Progress in Optics, E. Wolf, ed., 34, ch. 4, pp. 333-402, Elsevier, Amsterdam, 1995.

[4] D. Hattery, M. Hassan, S. Demos, and A. Gandjbakhche, "Hyperspectral Imaging of Kaposi's Sarcoma for Disease Assessment and Treatment Monitoring," in Proceedings of the IEEE Computer Society, 31 st Applied Imagery and Pattern Recognition Workshop, D. H. Schaefer, ed., pp. 124-132, 2002. 